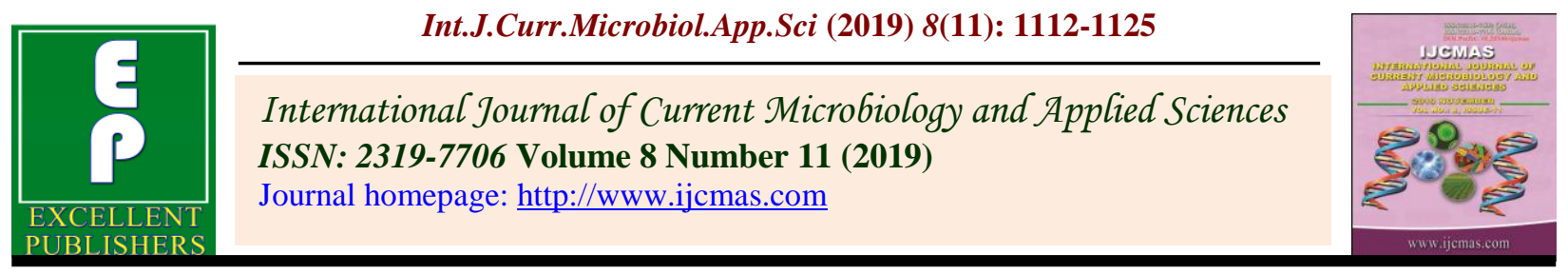

Original Research Article

https://doi.org/10.20546/ijcmas.2019.811.131

\title{
Effect of Novel Insecticide Molecules in Mulberry on Larval Parameters of Silkworm Bombyx mori L.
}

\author{
M.P. Yeshika ${ }^{1^{*}}$, K.G. Banuprakash ${ }^{1}$, K. Muruli Mohan ${ }^{2}$ and K.S. Vinoda ${ }^{3}$ \\ ${ }^{1}$ Department of Sericulture, UAS, GKVK, Bangalore - 560065, Karnataka, India \\ ${ }^{2}$ Department of Agricultural Entomology, UAS, GKVK, Bangalore - 560065, \\ Karnataka, India \\ ${ }^{3}$ Department of Silkworm Genetics and Breeding, College of Chintamani-563125, India \\ *Corresponding author
}

\section{A B S T R A C T}

Keywords

Mulberry,

Silkworm,

Dinotefuron,

Flonicamid,

Azadiractin, DDVP

Article Info

Accepted:

10 October 2019

Available Online:

10 November 2019
Novel insecticides with unique mode of action, were treated to mulberry leaves by foliar spray, later the leaves were fed to the silkworms which were reared on 10,20, 30 and 40 Days After Spraying (DAS) of insecticides to mulberry plants. Flonicamid 50 WG @ $0.3 \mathrm{~g} / \mathrm{l}$ recorded 100 per cent silkworm mortality and zero per cent mortality was recorded in all the other treatments. The prolongation of larval periods were noticed when the silkworms were fed with insecticide treated leaves at third, fourth and fifth instars. The silkworms recorded lowest larval weight in the treatment flonicamid @ $0.3 \mathrm{~g} / \mathrm{l}$ and highest larval weight was recorded in dinotefuran 20 SG@0.25 g/l and dichlorvos 76 EC @ $2.63 \mathrm{ml} / 1$ at 10, 20 30, 40 DAS. Effective Rate of Rearing of 100 per cent was recorded in silkworms fed on mulberry leaves from treatments pymetrozine $50 \mathrm{WG} @ 0.3 \mathrm{~g} / \mathrm{l}$ at $10 \mathrm{DAS}$, azadirachtin1\%@2 ml/1 and dichlorvos 76 EC @ $2.63 \mathrm{ml} / \mathrm{l}$ at 30 DAS.

\section{Introduction}

Sericulture is an integral part of the rural economy in an agrarian country like India. India is the second largest silk producing country next only to China. Mulberry is the sole food of silkworm, Bombyx mori L. and is infested by several pests. These pests affect the growth of mulberry and cause considerable damage to the plant and loss in the yield. The insecticides applied for the management of mulberry pests have greater impact on silkworm. Pesticides leave residues on mulberry leaves which have deleterious effect on the sensitive silkworm. To overcome this problem, safe waiting period should be followed for leaf harvest (Yokoyama, 1962). Field observations in India indicated loss of 
cocoon yield from silkworms fed on mulberry leaves sprayed with insecticides (Narasimhanna, 1988).

The residue of pesticides in the mulberry leaves could affect growth and quality of economic characteristics of cocoons. Sik et al., (1976) reported that more than 1.4 per cent of yield reduction in sericulture was due to the side effect of pesticide application, 49.4 per cent was due to the application of different pesticides in rice fields, 21.2 per cent in fruit gardens and 12.3 per cent in olericulture. Pest management in mulberry sericulture is absolutely selective because silkworm cannot tolerate even sub lethal doses of toxic compounds.

Dichlorvos is the recommended insecticide used for management of sucking pests in mulberry (Dandin et al., 2003). Newer chemicals with novel mode of action have been introduced for management of sucking pests in other crops viz., cotton, grapes, chilli, sunflower etc. However, the choices of newer insecticides require information against sucking insects in mulberry ecosystem, waiting period and residual toxicity. In the light of above, a study was initiated using different novel insecticides, corresponding waiting periods and residual toxicity to silkworm, especially on the larval parameters of silkworm, B mori L.

\section{Materials and Methods}

\section{Experimental layout}

The experiment was conducted at the Department of Sericulture, UAS, GKVK, Bengaluru with well established mulberry garden of $\mathrm{V}_{1}$ variety fed to Kolar Gold the commercial cross breed $\left(\mathrm{PM} \times \mathrm{CSR}_{2}\right)$. The experiment was laid out in Completely Randomized Design with 13 treatments, each replicated three times.

\section{Treatment details}

Insecticides like buprofezin 25 SC @ (1 ml/l and $2 \mathrm{ml} / \mathrm{l})$, pymetrozine $50 \mathrm{WG} @(0.3 \mathrm{~g} / \mathrm{l}$ and $0.6 \mathrm{~g} / \mathrm{l})$, flonicamid $50 \mathrm{WG} @(0.15 \mathrm{~g} / \mathrm{l}$ and $0.3 \mathrm{~g} / \mathrm{l})$, dinotefuron $20 \mathrm{SG} @(0.12 \mathrm{~g} / \mathrm{l}$ and $0.25 \mathrm{~g} / \mathrm{l})$, azadirachtin $1 \%$ @ (1 ml/1 and 2 $\mathrm{ml} / \mathrm{l})$, dichlorvos 76 EC @ (1.32 ml/l and 2.63 $\mathrm{ml} / \mathrm{l}$ ) and untreated control were sprayed on the mulberry plants and care was taken by holding a polythene cover along the treated plants while spraying to ensure that there was no drifting of chemicals due to wind and the treated leaves were fed to the silkworms.

Before the commencement of silkworm rearing the appliances were sun dried and rearing room, appliances were thoroughly cleaned and the floor was washed using 2 per cent bleaching powder solution. Then the entire rearing room was later disinfected by following standard procedure (Dandin et al., 2003). The rearing room was kept air tight for 24 hours and then the room was kept open and used for rearing. The third instar larvae were provided with chopped mulberry leaves of required quantity and quality. After 30 minutes of initial feeding, 90 larvae were transferred to each experimental tray in thee replications along with the mulberry leaves. Later, in order to assess extent of toxicity in insecticides to silkworm and to determine the safety period for effective molecules, the silkworm rearing was carried out. Observations such as larval instar duration (h), larval mortality (\%), matured larval weight (g) and ERR (\%) were recorded during rearing.

\section{Results and Discussion}

\section{Larval mortality (\%)}

The silkworm larval mortality was recorded from batch of worms fed with treated leaved harvested 10 days after spraying showed variation in the mortality rates among the 
treatments. Flonicamid 50 WG @ 0.3 g/l recorded 100 per cent silkworm larval mortality. However, in all other treatments including flonicamid 50 WG @ 0.15 g/l, no larval mortality was recorded.

No mortality was recorded in all the treatments including Flonicamid 50 WG @ $0.3 \mathrm{~g} / 1$ when silkworms were reared with the leaves harvested 20, 30 and 40 days after spraying (Table 1; Fig. 1).

Flonicamid insecticide was toxic to silkworms only when used at $0.3 \mathrm{~g} / 1$. However, at lower concentration of $0.15 \mathrm{~g} / 1$ it was found safer. Though flonicamid is a selective feeding blocker specific to sucking insects with repellant action, it appears that silkworms have lower tolerance limits for this molecule.

Other studies have revealed that insecticides belonging organophosphates produce toxic symptoms on silkworms when sprayed on mulberry plant. Ma Hui et al., (2006) reported toxicity of diazinon, dichlorvos, phoxim and triazophos on silkworms when plants were treated with these insecticides (Ma Hui et al., 2006). Similarly, residual toxicity of dimethoate and dichlorvos insecticides on silkworms was also reported by $\mathrm{Li}$ et al., (1995).

In the present investigation, barring flonicamid, all the new insecticide molecules were found safer on silkworms. The toxicity was not noticed even at 10 DAS. This clearly suggests that silkworms were not affected by the application of these insecticide molecules. The new molecules that were tried in this study possess unique modes of action on insects. They are highly selective on homopteron insects including mealybugs and have limited action on biting \& chewing type of insects (Gosh et al., 2014). When efficacy of insecticides and botanicals were used for regulating whitefly incidence on mulberry, treatments with neem oil @ 1500 ppm and azadirachtin $1 \%$ recorded the lowest mortality of silkworms with $10.8 \%$ and $11.6 \%$ at 7 DAS, $6.3 \%$ \& $8.3 \% 14$ DAS, respectively (Bandyopadhyay et al., 2013).

\section{Effect of insecticides on larval duration (h) in 10 days after spraying}

The silkworms reared on leaves harvested at 10 DAS of insecticide application exhibited significant difference among treatments.

At third instar, longest larval duration was recorded in the treatment flonicamid $50 \mathrm{WG}$ @ $0.3 \mathrm{~g} / \mathrm{l}(84.70 \mathrm{~h})$ followed by buprofezin 25 SC @ 2 ml/1 (84.37 h), dichlorvos 76 EC @ $1.32 \mathrm{ml} / \mathrm{l}(83.97 \mathrm{~h})$, azadirachtin $1 \%$ @ $1 \mathrm{ml} / \mathrm{l}$ $(83.33 \mathrm{~h})$ and dinotefuron $50 \mathrm{SG} @ 0.12 \mathrm{~g} / \mathrm{l}$ $(79.13 \mathrm{~h})$ which were statistically on par with one another. The shortest larval duration of third instar was observed in the treatment azardirachtin 1\%@2 ml/l (62.67 h) and dinotefuron 50 SG @ 0.25 g/l (66.43 h) (Table 2).

Larval instar duration recorded at fourth instar indicated significant difference among the treatments. Longest fourth instar larval duration of silkworms in the treatment buprofezin 25 SC @ 2 ml/l (123.47 h), along with dichlorvos 76 EC @ 1.32 ml/l (123.00 h) and azadirachtin 1\% @ 1 ml/l (118.17 h) which were on par with each other.

Lowest larval duration at fourth instar was recorded in the treatment pymetrozine $50 \mathrm{WG}$ @ $0.6 \mathrm{~g} / \mathrm{l}(89.07 \mathrm{~h})$ and it was statistically on par with dinotefuron 50 SG @ 0.25 g/l (89.67 h), azardirachtin 1\% @ 2 ml/l (90.67 h), dichlorvos 76 EC @ 2.63 ml/l (93.67 h) and untreated control of about $96.17 \mathrm{~h}$. Flonicamid 50 WG@0.3 g/l treated silkworms exhibited cent per cent mortality, due to which it wasn't possible to calculate the larval mortality (Table 2). 
The silkworms reared with azadirachtin $1 \%$ @ $1 \mathrm{ml} / \mathrm{l}$ exhibited the longest larval duration of V instar $(221.83 \mathrm{~h})$, followed by flonicamid 50 WG@0.15 g/l which recorded the larval duration of $220.40 \mathrm{~h}$, it was followed by pymetrozine 50 WG @ $0.3 \mathrm{~g} / \mathrm{l}(220.10 \mathrm{~h})$, buprofezin 25 SC @ 2 ml/l $(214.53$ h) and dichlorvos 76 EC @ 1.32 ml/l (214 h) which were on par with each other. Shortest larval duration at fifth instar was recorded in the treatment dichlorvos 76 EC @ 2.63 ml/1 $(195.37 \mathrm{~h})$ and it was statistically on par with azadirachtin 1 \% @ 2ml/1 (195.67 h), dinotefuron 20 SG @ $0.25 \mathrm{~g} / 1$ (197.45 h), untreated control (199.25 h), buprofezin $25 \mathrm{SC}$ @ 1ml/1 (204.37 h) and pymetrozine $50 \mathrm{WG}$ @ 0.6 g/l (207.43 h). Flonicamid 50 WG @ $0.3 \mathrm{~g} / \mathrm{l}$ exhibited nil value for larval duration among the treatments (Table 2).

\section{Effect of insecticides on larval duration (h) in 20 days after spraying}

The silkworm larval duration (h) 20 DAS showed significant difference among the treatments. The longest larval duration of third instar was recorded in the treatment azadirachtin 1\% @ $1 \mathrm{ml} / \mathrm{l}(89.23 \mathrm{~h})$. The shortest larval duration was recorded in pymetrozine 50WG @ $0.6 \mathrm{~g} / \mathrm{l}$ (71.60 h) which was statistically on par with dichlorvos 76 EC @ $2.63 \mathrm{ml} / \mathrm{l}(71.80 \mathrm{~h})$ and untreated control (73.29 h).

The treatment buprofezin 25 SC @ $2 \mathrm{ml} / 1$ exhibited longest fourth instar larval duration of $123.77 \mathrm{~h}$ which was statistically on par with buprofezin 25 SC @ $2 \mathrm{ml} / \mathrm{l}(122.80 \mathrm{~h})$ and flonicamid 50WG@0.15 g/l (120.70 h).

Pymetrozine 50 WG @ 0.3 g/l also exhibited longer larval duration of $117.83 \mathrm{~h}$ which was statistically on par with azadirachtin 1\% @ 1 $\mathrm{ml} / \mathrm{l}(116.30 \mathrm{~h})$. The shortest larval duration was recorded in dinotefuron 20 SG @ $0.25 \mathrm{~g} / \mathrm{l}$ (90.03 h) (Table 3).
Fifth instar silkworms exhibited longest larval duration in azadirachtin 1\%@1 ml/l (221 h) which recorded statistically on par larval duration with dichlorvos 76 EC @ 1.32 ml/l (217.60 h), buprofezin 25 SC @ $2 \mathrm{ml} / \mathrm{l}$ (215.87 h), pymetrozine 50 WG @0.3 g/l (207.47 h) and flonicamid 50 WG @0.3 g/l (205.83 h). Flonicamid 50 WG @0.15 g/l exhibited shortest fifth instar larval duration $(171.10 \mathrm{~h})$ which was statistically on par with dichlorvos 76 EC @ 2.63 ml/l (190 h) (Table 3).

\section{Effect of insecticides on larval duration (h) in 30 days after spraying}

The silkworm larval duration recorded when the worms fed with insecticide treated mulberry during 30 DAS recorded significant difference among the treatments.

Third instar silkworm larvae exhibited longest larval duration of $89.23 \mathrm{~h}$ in azadirachtin $1 \%$ @ 1 ml/l. Dichlorvos 76 EC @ 1.32 ml/l also recorded higher larval duration of $85 \mathrm{~h}$ which was statistically on par with buprofezin $25 \mathrm{SC}$ @ $2 \mathrm{ml} / \mathrm{l}(82.37 \mathrm{~h})$. The shortest larval duration was exhibited by pymetrozine $50 \mathrm{WG}$ @ $0.6 \mathrm{~g} / \mathrm{l}(71.60 \mathrm{~h})$ which was on par with dichlorvos 76 EC @ 2.63 ml/1 (71.80 h) and untreated control (73.29 h) (Table 4).

Pymetrozine 50 WG @ 0.3 g/l recorded longest fourth instar larval duration of 119.23 $\mathrm{h}$ which was statistically on par with flonicamid 50 WG @ $0.3 \mathrm{~g} / \mathrm{l}(116.10 \mathrm{~h})$, azadirachtin 1\%@1 ml/l $(115.03 \mathrm{~h})$ and flonicamid 50 WG @0.15 g/l (114.63 h). The shortest fourth instar larval duration was recorded in dinotefuron 20 SG @ $0.25 \mathrm{~g} / \mathrm{l}$ (85.97 h) (Table 4).

The longest fifth instar larval duration was exhibited in the treatment dichlorvos76 EC @ $1.32 \mathrm{ml} / \mathrm{l}(221.47 \mathrm{~h})$ which was on par with azadirachtin $1 \%$ @ $\quad 1 \quad \mathrm{ml} / 1$ (218.03 h). 
Flonicamid 50 WG @ 0.3 g/l (212.07 h) exhibited statistically on par larval duration with pymetrozine 50 WG @ 0.3 g/l (210.77 h), buprofezin $25 \mathrm{SC} @ 2 \mathrm{ml} / \mathrm{l}(210 \mathrm{~h})$ and flonicamid 50 WG @ $0.15 \mathrm{~g} / \mathrm{l}$ (207.80 h). The shortest larval duration was recorded in untreated control (187.67 h). Dichlorvos 76 EC@2.63 ml/l (190.80 h), dinotefuron 20 SG @ $0.25 \mathrm{~g} / \mathrm{l}(194.77 \mathrm{~h})$ and azadirachtin 1\% @ $2 \mathrm{ml} / \mathrm{l}(194.33 \mathrm{~h})$ exhibited on par values (Table 4).

\section{Effect of insecticides on larval duration (h) in 40 days after spraying}

The silkworm larval duration in the batches reared with mulberry sprayed with insecticides 40 DAS recorded significant difference among the treatments.

The longest third instar larval duration was exhibited by pymetrozine 50 WG @ $0.3 \mathrm{~g} / 1$ $(95.80 \mathrm{~h})$ which was on par with buprofezin 25 SC @ 2 ml/l (93.33 h). Azadirachtin $1 \%$ @ $1 \mathrm{ml} / \mathrm{l}$ also recorded longer larval duration of $89.43 \mathrm{~h}$ which was statistically on par with buprofezin 25 SC @ $1 \mathrm{ml} / \mathrm{l}(85.77 \mathrm{~h})$ and dichlorvos 76 EC @ 1.32 ml/l (84.60 h). Untreated control exhibited the shortest third instar larval duration of $74.53 \mathrm{~h}$ which exhibited statistically on par values with azadirachtin $1 \%$ @ $2 \mathrm{ml} / \mathrm{l} \quad(74.57 \mathrm{~h})$, dichlorvos 76 EC @ 2.63 ml/l (74.94 h), dinotefuron 20 SG @ $0.25 \mathrm{~g} / \mathrm{l}(75.80 \mathrm{~h})$, pymetrozine 50 WG @ $0.6 \mathrm{~g} / \mathrm{l}(76.63 \mathrm{~h})$ and dinotefuron 20 SG @0.12 g/l (78.13 h) (Table $5)$.

Fourth instar larvae exhibited longest larval duration in the treatment pymetrozine $50 \mathrm{WG}$ @ $0.3 \mathrm{~g} / 1122.42 \mathrm{~h}$ which was statistically on par with azadirachtin 1\%@1 ml/1 116.83 h. Dichlorvos 76 EC @ $1.32 \mathrm{ml} /$ also exhibited longer larval duration of $116.33 \mathrm{~h}$. The shortest larval duration was recorded in dinotefuron 20 SG @ 0.25 g/l 87.40 h which was statistically on par with azadirachtin $1 \%$ @ $2 \mathrm{ml} / 189.43 \mathrm{~h}$ (Table 5).

Azadirachtin 1\% @ $1 \mathrm{ml} / \mathrm{l}$ exhibited the longest larval duration of fifth instar silkworm larvae $(217 \mathrm{~h})$ which was statistically on par with flonicamid 50 WG @ 0.15 g/l (215.97 h), dichlorvos 76 EC @ $1.32 \mathrm{ml} / 1$ (213.63 h) and flonicamid 50 WG @0.15 g/l (212 h). The shortest fifth instar larval duration was exhibited by buprofezin 25 SC @ 1 ml/l (105.22 h) (Table 5).

The third instar larval duration was prolonged to $84.70 \& 84.37 \mathrm{~h}$ when the worms were fed on mulberry leaves sprayed with flonicamid 50WG@0.3 g/l, buprofezin 25 SC @ 2 ml/1, respectively when worms were fed with leaves harvested 10DAS. In the silkworm batches reared on leaves at 10 days after the spraying of insecticides, the shortest larval duration of $62.67 \mathrm{~h}$ in third instar, $90.67 \mathrm{~h}$ in fourth instar and $195.67 \mathrm{~h}$ in fifth instar was observed in the treatment dinotefuron 50SG @ $0.25 \mathrm{~g} / \mathrm{l}$ followed by dichlorvos 76 EC @ $2.63 \mathrm{ml} / 1$ (73.50 $\mathrm{h}$ in third instar, $93.67 \mathrm{~h}$ in fourth instar and 195. $37 \mathrm{~h}$ in fifth instar). At 20 DAS, azadirachtin 1\%@1 ml/ 1 and buprofezin 25 SC@ 2 ml/l recorded longer larval durations as compared to untreated control at different instars. At 30 DAS, prolonged larval duration was noticed in dichlorvos, pymetrozine, buprofezin treated plants. The longest third and fourth instar larval duration was exhibited by pymetrozine $50 \mathrm{WG} @ 0.3 \mathrm{~g} / \mathrm{l}(95.80 \mathrm{~h})$ at 40 DAS rearing.

Similarly, Kumutha et al., (2009, 2013) also noticed the increased larval and pupal duration with increase in insecticide concentration of dichlorvos $76 \%$ EC and azadirachtin 300 ppm, neem oil and methyl parathion. Narayanawamy et al., (2017) also reported increasing silkworm larval duration in fifth instar after the application of NSKE $4 \%$ on mulberry. 
Table.1 Effect of insecticides treated mulberry on silkworm mortality (\%) reared on leaves harvested at different days after spraying

\begin{tabular}{|c|c|c|c|c|c|}
\hline \multirow{2}{*}{\multicolumn{2}{|c|}{ Treatments }} & \multicolumn{4}{|c|}{ Silkworm larval mortality (\%) } \\
\hline & & 10 DAS & 20 DAS & 30 DAS & 40 DAS \\
\hline$T_{1}$ & Buprofezin 25 SC @ 1ml/1 & 0 & 0 & 0 & 0 \\
\hline $\mathbf{T}_{2}$ & Buprofezin 25 SC @ $2 \mathrm{ml} / \mathrm{l}$ & 0 & 0 & 0 & 0 \\
\hline $\mathbf{T}_{3}$ & Pymetrozine 50WG @0.3 g/l & 0 & 0 & 0 & 0 \\
\hline $\mathbf{T}_{4}$ & Pymetrozine 50WG @0.6g/1 & 0 & 0 & 0 & 0 \\
\hline $\mathbf{T}_{5}$ & Flonicamid 50 WG @0.15 g/l & 0 & 0 & 0 & 0 \\
\hline $\mathbf{T}_{6}$ & Flonicamid 50 WG @ $0.3 \mathrm{~g} / 1$ & 100 & 0 & 0 & 0 \\
\hline $\mathbf{T}_{7}$ & Dinotefuron 20 SG @ $0.12 \mathrm{~g} / \mathrm{l}$ & 0 & 0 & 0 & 0 \\
\hline $\mathbf{T}_{8}$ & Dinotefuron20 SG @0.25 g/l & 0 & 0 & 0 & 0 \\
\hline $\mathbf{T}_{9}$ & Azadirachtin 1\%@1 ml/l. & 0 & 0 & 0 & 0 \\
\hline$T_{10}$ & Azadirachtin 1\%@2 ml/1 & 0 & 0 & 0 & 0 \\
\hline$T_{11}$ & Dichlorvos 76 EC @ 1.32 ml/l & 0 & 0 & 0 & 0 \\
\hline $\mathbf{T}_{12}$ & $\begin{array}{l}\text { Dichlorvos } 76 \text { EC @ } 2.63 \text { ml/l (std. } \\
\text { check) }\end{array}$ & 0 & 0 & 0 & 0 \\
\hline $\mathbf{T}_{13}$ & Untreated control & 0 & 0 & 0 & 0 \\
\hline
\end{tabular}

DAS- Days after spraying.

Table.2 Effect of insecticides on larval duration (h) 10 days after spraying

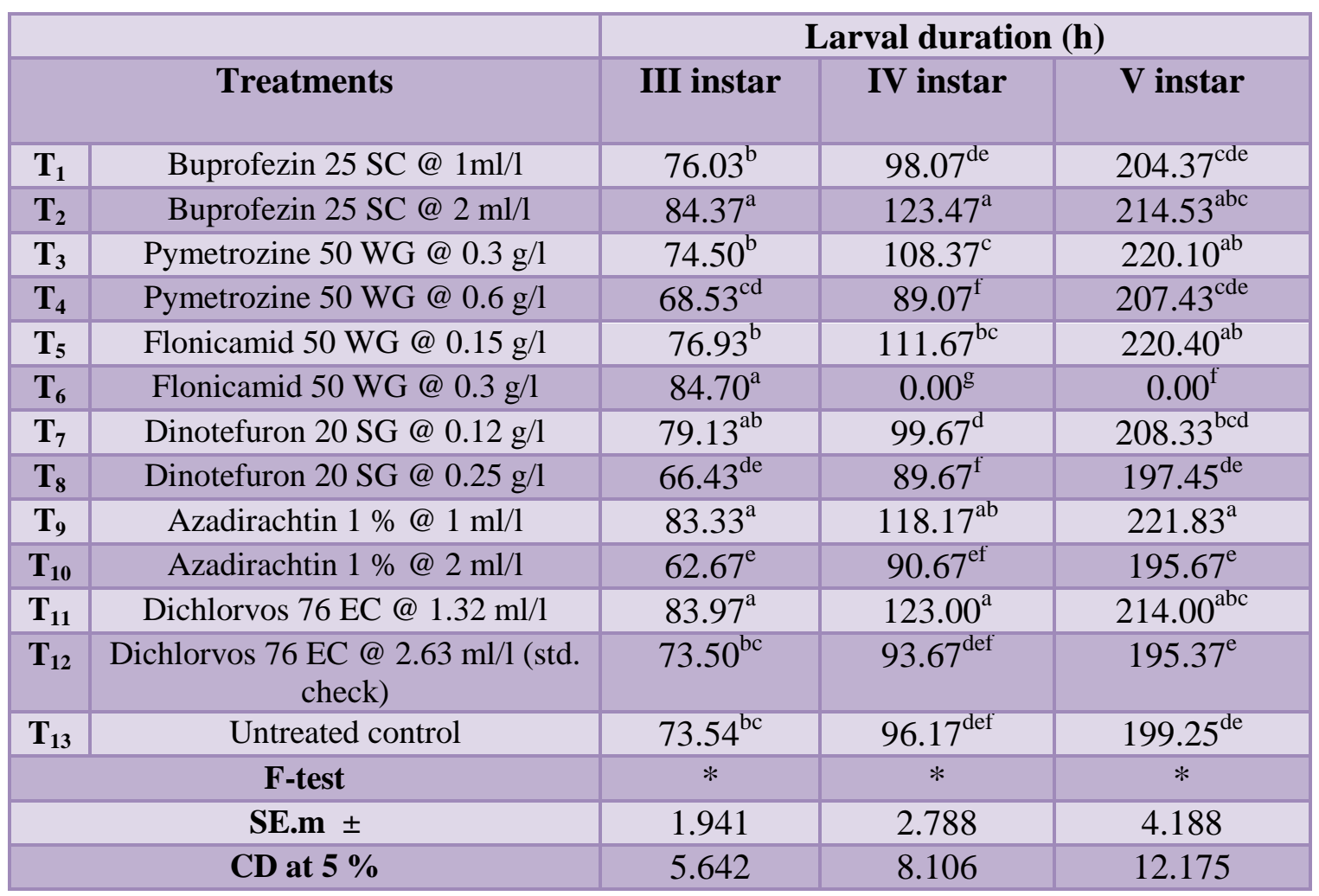

*Significant at $5 \%$, DAS - Days after spraying 
Table.3 Effect of insecticides on larval duration (h) 20 days after spraying

\begin{tabular}{|c|l|c|c|c|}
\hline \multicolumn{2}{|c|}{ Treatments } & \multicolumn{3}{c|}{ Larval duration (h) } \\
\cline { 2 - 5 } & III instar & IV instar & V instar \\
\hline $\mathbf{T}_{\mathbf{1}}$ & Buprofezin 25 SC @ 1 1 //1 & $81.57^{\mathrm{c}}$ & $108.10^{\mathrm{d}}$ & 198.33 \\
\hline $\mathbf{T}_{\mathbf{2}}$ & Buprofezin 25 SC @ 2 ml/1 & $82.03^{\mathrm{bc}}$ & $123.77^{\mathrm{a}}$ & 215.87 \\
\hline $\mathbf{T}_{\mathbf{3}}$ & Pymetrozine 50 WG @ 0.3 g/l & $77.17^{\mathrm{de}}$ & $117.83^{\mathrm{bc}}$ & 207.47 \\
\hline $\mathbf{T}_{\mathbf{4}}$ & Pymetrozine 50 WG @ 0.6 g/l & $71.60^{\mathrm{g}}$ & $95.33^{\mathrm{f}}$ & 195.40 \\
\hline $\mathbf{T}_{\mathbf{5}}$ & Flonicamid 50 WG @ 0.15 g/l & $81.20^{\mathrm{c}}$ & $120.70^{\mathrm{ab}}$ & 171.10 \\
\hline $\mathbf{T}_{\mathbf{6}}$ & Flonicamid 50 WG @ 0.3 g/l & $81.30^{\mathrm{c}}$ & $122.80^{\mathrm{a}}$ & 205.83 \\
\hline $\mathbf{T}_{\mathbf{7}}$ & Dinotefuron 20 SG @ 0.12 g/l & $79.53^{\mathrm{cd}}$ & $101.97^{\mathrm{e}}$ & 199.77 \\
\hline $\mathbf{T}_{\mathbf{8}}$ & Dinotefuron 20 SG @ 0.25 g/l & $75.20^{\mathrm{ef}}$ & $90.03^{\mathrm{g}}$ & 193.10 \\
\hline $\mathbf{T}_{\mathbf{9}}$ & Azadirachtin 1 \% @ 1 ml/1 & $89.23^{\mathrm{a}}$ & $116.20^{\mathrm{c}}$ & 221.00 \\
\hline $\mathbf{T}_{\mathbf{1 0}}$ & Azadirachtin 1 \% @ 2 ml/1 & $74.47^{\mathrm{egf}}$ & $94.00^{\mathrm{f}}$ & 190.80 \\
\hline $\mathbf{T}_{\mathbf{1 1}}$ & Dichlorvos 76 EC @ 1.32 ml/1 & $85.00^{\mathrm{b}}$ & $110.06^{\mathrm{d}}$ & 217.60 \\
\hline $\mathbf{T}_{\mathbf{1 2}}$ & $\begin{array}{l}\text { Dichlorvos 76 EC @ 2.63 ml/1 } \\
\text { (std. check) }\end{array}$ & $71.80^{\mathrm{g}}$ & $95.10^{\mathrm{f}}$ & 190.00 \\
\hline $\mathbf{T}_{\mathbf{1 3}}$ & Untreated control & $73.29^{\mathrm{fg}}$ & $97.30^{\mathrm{f}}$ & 197.51 \\
\hline & F-test & $*$ & $*$ & NS \\
\hline & SE.m \pm & 1.025 & 1.298 & 9.614 \\
\hline
\end{tabular}

*Significant at $5 \%$, DAS - Days after spraying.

Table.4 Effect of insecticides on larval duration (h) 30days after spraying

\begin{tabular}{|c|c|c|c|c|}
\hline \multirow{2}{*}{\multicolumn{2}{|c|}{ Treatments }} & \multirow{2}{*}{\multicolumn{3}{|c|}{ Larval duration (h) }} \\
\hline & & & & \\
\hline $\mathbf{T}_{1}$ & Buprofezin25 SC @ 1ml/1 & $\begin{array}{c}\text { III instar } \\
81.90^{c}\end{array}$ & $\begin{array}{l}\text { IV instar } \\
102.80^{\mathrm{de}}\end{array}$ & $\begin{array}{l}\text { V instar } \\
203.73^{\text {de }}\end{array}$ \\
\hline $\mathbf{T}_{2}$ & Buprofezin 25 SC @ 2 ml/1 & $82.37^{\mathrm{bc}}$ & $109.70^{\mathrm{bc}}$ & $210.00^{\text {cd }}$ \\
\hline $\mathbf{T}_{\mathbf{3}}$ & Pymetrozine 50 WG @0.3 g/l & $77.17^{\mathrm{de}}$ & $119.23^{\mathrm{a}}$ & $210.77^{\mathrm{bcc}}$ \\
\hline $\mathbf{T}_{4}$ & Pymetrozine 50 WG @0.6 g/l & $71.60^{g}$ & $86.90^{\text {gh }}$ & $195.33^{f}$ \\
\hline $\mathbf{T}_{5}$ & Flonicamid 50 WG @ $0.15 \mathrm{~g} / 1$ & $80.63^{c}$ & $114.63^{a b c}$ & $207.80^{\text {cd }}$ \\
\hline $\mathbf{T}_{6}$ & Flonicamid 50 WG @ $0.3 \mathrm{~g} / \mathrm{l}$ & $81.93^{c}$ & $116.10^{\mathrm{ab}}$ & $212.07^{\mathrm{bc}}$ \\
\hline $\mathbf{T}_{7}$ & Dinotefuron 20 SG @ 0.12 g/l & $79.53^{\text {cd }}$ & $91.90^{\text {fgh }}$ & $198.00^{\mathrm{ef}}$ \\
\hline $\mathbf{T}_{8}$ & Dinotefuron20 SG @ 0.25 g/l & $75.87^{\mathrm{ef}}$ & $85.97^{\mathrm{h}}$ & $194.77^{\mathrm{fg}}$ \\
\hline $\mathbf{T}_{9}$ & Azadirachtin 1\%@1 ml/1 & $89.23^{\mathrm{a}}$ & $115.03^{a b c}$ & $218.03^{\mathrm{ab}}$ \\
\hline $\mathbf{T}_{10}$ & Azadirachtin 1\%@2 ml/l & $75.13^{\mathrm{ef}}$ & $89.83^{\text {gh }}$ & $194.33^{\mathrm{fg}}$ \\
\hline $\mathbf{T}_{11}$ & Dichlorvos 76 EC @ 1.32 ml/1 & $85.00^{\mathrm{b}}$ & $108.77^{\mathrm{cd}}$ & $221.47^{\mathrm{a}}$ \\
\hline $\mathbf{T}_{12}$ & $\begin{array}{l}\text { Dichlorvos } 76 \text { EC @ } 2.63 \text { ml/1 } \\
\text { (std. check) }\end{array}$ & $71.80^{\mathrm{g}}$ & $93.67^{\mathrm{fg}}$ & $190.80^{\mathrm{fg}}$ \\
\hline $\mathbf{T}_{13}$ & Untreated control & $73.29^{\mathrm{fg}}$ & $98.03^{\mathrm{ef}}$ & $187.67^{\mathrm{g}}$ \\
\hline & F-test & $*$ & $*$ & $*$ \\
\hline & SE.m \pm & 1.012 & 2.341 & 2.594 \\
\hline & CD at $5 \%$ & 2.943 & 6.804 & 7.541 \\
\hline
\end{tabular}

*Significant at $5 \%$, DAS - Days after spraying. 
Table.5 Effect of insecticides on larval duration (h) 40 days after spraying

\begin{tabular}{|c|c|c|c|c|}
\hline \multirow{2}{*}{\multicolumn{2}{|c|}{ Treatments }} & \multicolumn{3}{|c|}{ Larval duration (h) } \\
\hline & & III instar & IV instar & $V$ instar \\
\hline $\mathbf{T}_{1}$ & Buprofezin 25 SC @1ml/1 & $85.77^{\text {cd }}$ & $95.53^{\mathrm{d}}$ & $105.22^{\mathrm{e}}$ \\
\hline $\mathbf{T}_{2}$ & Buprofezin 25 SC @ 2 ml/1 & $93.33^{\mathrm{ab}}$ & $109.92^{\mathrm{c}}$ & $203.64^{\mathrm{b}}$ \\
\hline $\mathbf{T}_{3}$ & Pymetrozine 50 WG @0.3 g/1 & $95.80^{\mathrm{a}}$ & $122.42^{\mathrm{a}}$ & $204.68^{b}$ \\
\hline $\mathbf{T}_{4}$ & Pymetrozine 50 WG @0.6g/1 & $76.63^{\mathrm{f}}$ & $89.57^{\mathrm{efg}}$ & $187.73^{\mathrm{d}}$ \\
\hline$T_{5}$ & Flonicamid50 WG @ $0.15 \mathrm{~g} / 1$ & $84.27^{\mathrm{d}}$ & $108.60^{\mathrm{c}}$ & $215.97 \mathrm{a}$ \\
\hline$T_{6}$ & Flonicamid 50 WG @ $0.3 \mathrm{~g} / 1$ & $83.00^{\mathrm{de}}$ & $108.83^{\mathrm{c}}$ & $212.00^{\mathrm{a}}$ \\
\hline $\mathbf{T}_{7}$ & Dinotefuron 20 SG @ $0.12 \mathrm{~g} / 1$ & $78.13^{\mathrm{ef}}$ & $95.20^{\text {de }}$ & $195.87^{c}$ \\
\hline $\mathbf{T}_{8}$ & Dinotefuron20 SG @ $0.25 \mathrm{~g} / \mathrm{l}$ & $75.80^{\mathrm{f}}$ & $87.40^{\mathrm{g}}$ & $191.37^{\text {cd }}$ \\
\hline$T_{9}$ & Azadirachtin 1\%@1 ml/l & $89.43^{b c}$ & $116.83^{\mathrm{ab}}$ & $217.00 \mathrm{a}$ \\
\hline $\mathbf{T}_{10}$ & Azadirachtin 1\%@2 ml/1 & $74.57^{\mathrm{f}}$ & $89.43^{\mathrm{fg}}$ & $189.6^{\mathrm{cd}}$ \\
\hline$T_{11}$ & Dichlorvos76 EC @ 1.32 ml/1 & $84.60^{\mathrm{cd}}$ & $116.33^{\mathrm{b}}$ & $213.63^{\mathrm{a}}$ \\
\hline $\mathbf{T}_{12}$ & Dichlorvos 76 EC @ $2.63 \mathrm{ml} / 1$ (std. check) & $74.94 \mathrm{f}$ & $91.60^{\operatorname{defg}}$ & $190.62^{\text {cd }}$ \\
\hline $\mathbf{T}_{13}$ & Untreated control & $74.53 \mathrm{f}$ & $95.00^{\operatorname{def}}$ & $194.00^{\text {cd }}$ \\
\hline & F-test & * & * & * \\
\hline & SE.m \pm & 1.718 & 1.974 & 2.236 \\
\hline & CD at $5 \%$ & 4.994 & 5.739 & 6.499 \\
\hline
\end{tabular}

*Significant at $5 \%$, DAS - Days after spraying.

Table.6 Effect of insecticides on larval weight ( $g /$ larva )

\begin{tabular}{|c|c|c|c|c|c|}
\hline \multicolumn{2}{|r|}{ Treatments } & 10 DAS & 20 DAS & 30 DAS & 40 DAS \\
\hline $\mathbf{T}_{1}$ & Buprofezin25 SC @1ml/1 & $2.34^{\mathrm{de}}$ & $2.74^{\mathrm{cd}}$ & $2.75^{\mathrm{cde}}$ & $2.57^{\mathrm{de}}$ \\
\hline $\mathbf{T}_{2}$ & Buprofezin 25 SC @ 2 ml/1 & $2.38^{\mathrm{de}}$ & $2.44^{\mathrm{ef}}$ & $2.26^{\mathrm{g}}$ & $2.45^{\mathrm{e}}$ \\
\hline $\mathbf{T}_{3}$ & Pymetrozine 50WG @0.3 g/l & $2.27^{\mathrm{e}}$ & $2.62^{\mathrm{de}}$ & $2.40^{\mathrm{tg}}$ & $2.53^{\mathrm{de}}$ \\
\hline $\mathbf{T}_{4}$ & Pymetrozine 50WG @0.6 g/l & $2.50^{\text {cde }}$ & $2.79^{\mathrm{cd}}$ & $2.89^{\mathrm{bc}}$ & $2.80^{\mathrm{bc}}$ \\
\hline$T_{5}$ & Flonicamid 50 WG @0.15 g/l & $2.30^{\mathrm{e}}$ & $2.73^{\mathrm{cd}}$ & $2.60^{\operatorname{det}}$ & $2.51^{\mathrm{de}}$ \\
\hline $\mathbf{T}_{6}$ & Flonicamid 50 WG @ $0.3 \mathrm{~g} / 1$ & $0.00^{\mathrm{f}}$ & $2.07^{\mathrm{g}}$ & $2.18^{\mathrm{g}}$ & $2.25^{\mathrm{f}}$ \\
\hline $\mathbf{T}_{7}$ & Dinotefuron20 SG @0.12 g/l & $2.69^{\mathrm{bc}}$ & $2.72^{\mathrm{cd}}$ & $2.81^{\mathrm{cd}}$ & $2.69^{\mathrm{cd}}$ \\
\hline $\mathbf{T}_{8}$ & Dinotefuron 20 SG @ $0.25 \mathrm{~g} / \mathrm{l}$ & $2.94^{\mathrm{ab}}$ & $3.09^{\mathrm{ab}}$ & $3.06^{\mathrm{ab}}$ & $3.05^{\mathrm{a}}$ \\
\hline$T_{9}$ & Azadirachtin1\%@1 ml/l & $2.57^{\mathrm{cd}}$ & $2.38^{\mathrm{f}}$ & $2.41 \mathrm{f}^{\mathrm{g}}$ & $2.65^{\text {cd }}$ \\
\hline $\mathbf{T}_{10}$ & Azadirachtin 1\%@2 ml/1 & $2.48^{\text {cde }}$ & $2.86^{\mathrm{c}}$ & $2.88^{\mathrm{bc}}$ & $2.94^{\mathrm{ab}}$ \\
\hline$T_{11}$ & Dichlorvos76 EC @ 1.32 ml/l & $2.25^{\mathrm{e}}$ & $2.58^{\text {def }}$ & $2.53^{\mathrm{ef}}$ & $2.45^{\mathrm{e}}$ \\
\hline $\mathbf{T}_{12}$ & $\begin{array}{l}\text { Dichlorvos } 76 \text { EC @ } 2.63 \text { ml/l. } \\
\text { (std. check) }\end{array}$ & $2.67^{c}$ & $2.95^{\mathrm{bc}}$ & $2.93^{\mathrm{abc}}$ & $2.93^{\mathrm{ab}}$ \\
\hline $\mathbf{T}_{13}$ & Untreated control & $3.01^{\mathrm{a}}$ & $3.20^{\mathrm{a}}$ & $3.15^{\mathrm{a}}$ & $3.10^{\mathrm{a}}$ \\
\hline & F-test & $*$ & * & $*$ & $*$ \\
\hline & SE.m \pm & 0.092 & 0.073 & 0.082 & 0.066 \\
\hline & CD at $5 \%$ & 0.212 & 0.212 & 0.239 & 0.191 \\
\hline
\end{tabular}

*Significant at $5 \%$, DAS - Days after spraying. 
Table.7 Effect of insecticides on Effective Rate of Rearing (ERR) (\%)

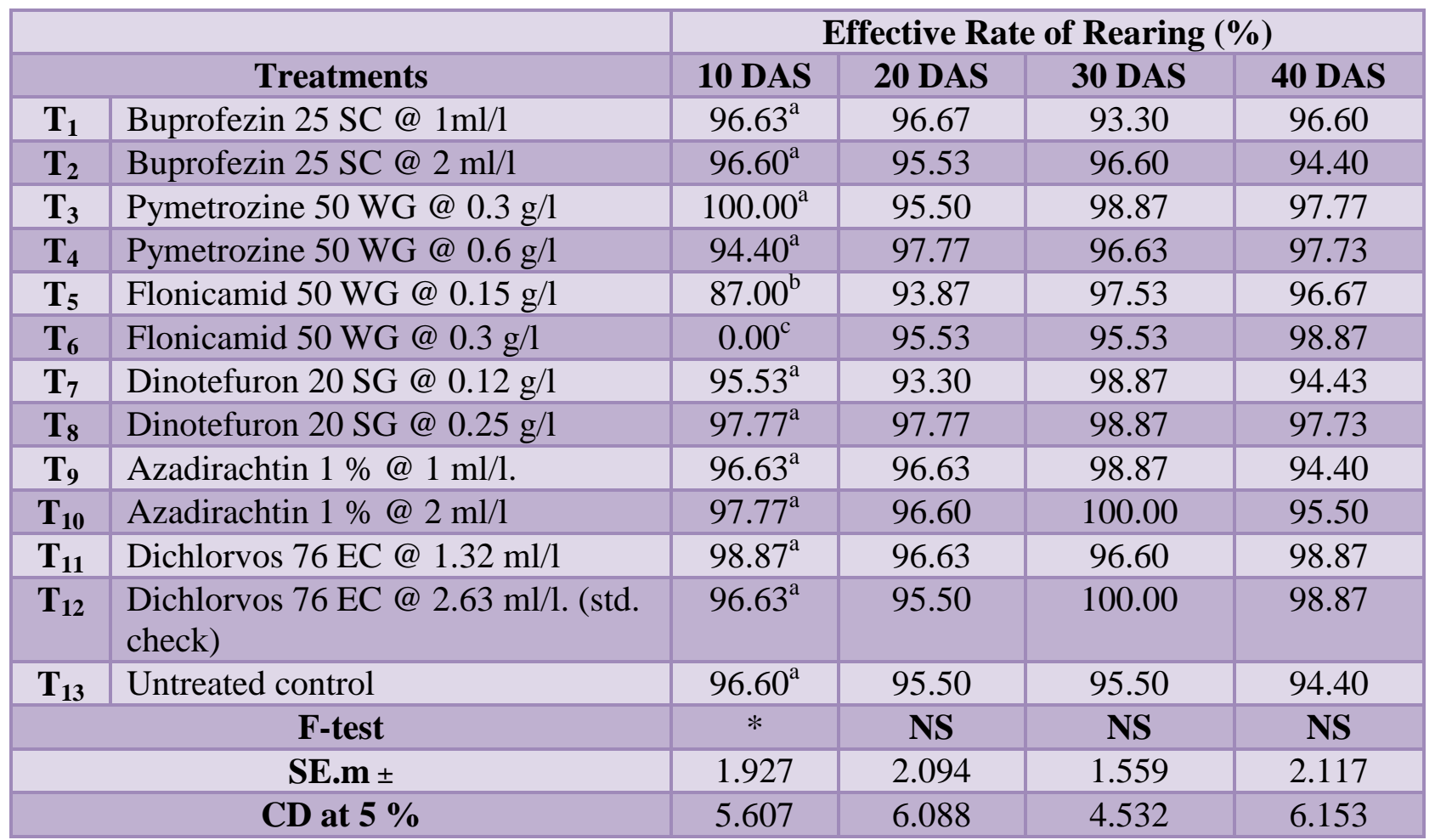

*Significant at $5 \%, \mathrm{NS}$ - Non significant, DAS - Days after spraying.

Plate.1 Third instar silkworm larval mortality in the treatment flonicamid 50 WG @0.3 g/l

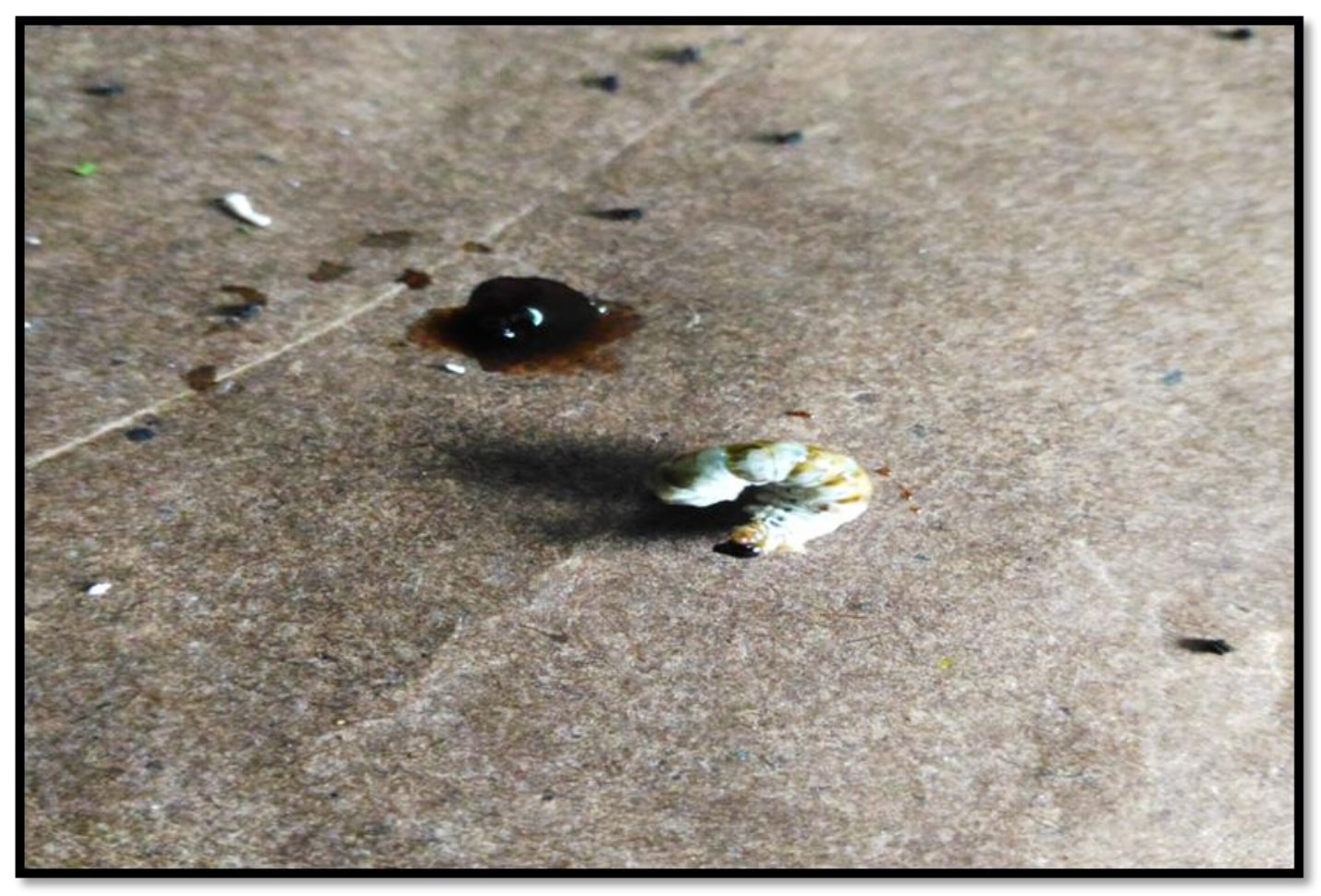


Plate.2 Third instar silkworm larvae in untreated control

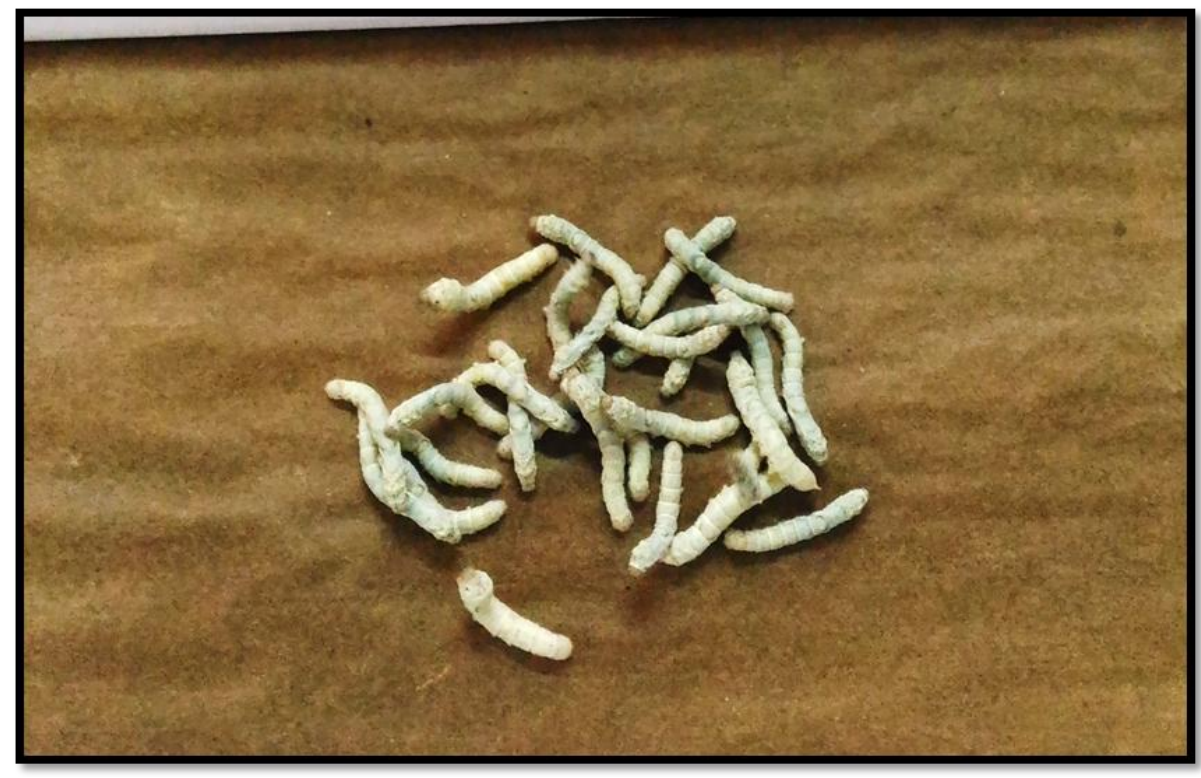

Fig.1 Effect of insecticides on larval mortality (\%) in the batches reared on 10 DAS, 20 DAS, 30 DAS and 40 DAS

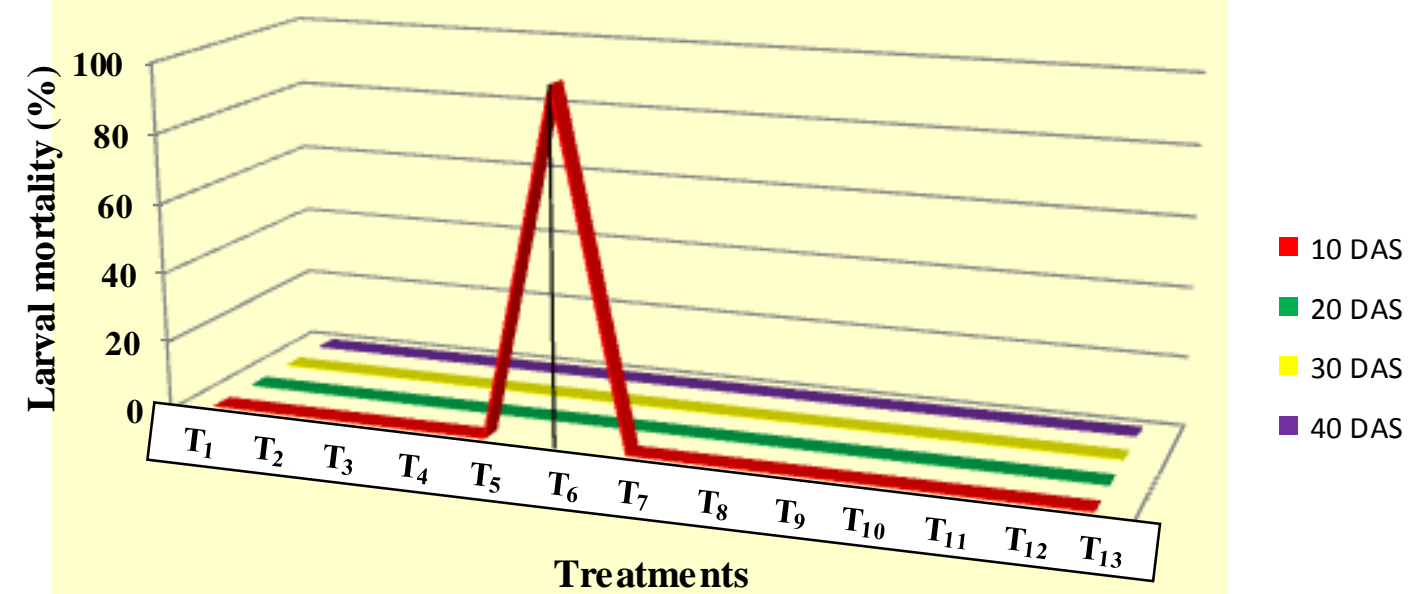

$\mathbf{T}_{1}$ - Buprofezin $25 \mathrm{SC} @ 1 \mathrm{ml} / 1$

$\mathbf{T}_{2^{-}}$Buprofezin $25 \mathrm{SC} @ 2 \mathrm{ml} / \mathrm{l}$

$\mathbf{T}_{3^{-}}$Pymetrozine $50 \mathrm{WG} @ 0.3 \mathrm{~g} / 1$

$\mathbf{T}_{4}$ - Pymetrozine 50 WG @ $0.6 \mathrm{~g} / \mathrm{l}$

$\mathbf{T}_{5^{-}}$Flonicamid $50 \mathrm{WG} @ 0.15 \mathrm{~g} / 1$

$\mathbf{T}_{6}$ - Flonicamid 50 WG @ $0.3 \mathrm{~g} / 1$

$\mathbf{T}_{7^{-}}$Dinotefuron 20 SG @ $0.12 \mathrm{~g} / \mathrm{l}$
$\mathbf{T}_{8^{-}}$Dinotefuron 20 SG @ $0.25 \mathrm{~g} / \mathrm{l}$

$\mathbf{T}_{\mathbf{9}}$ - Azadirachtin 1\%@1 ml/1

$\mathbf{T}_{10}$-Azadirachtin 1\%@2 ml/l

$\mathbf{T}_{11}$ - Dichlorovas 76 EC @ $1.32 \mathrm{ml} / 1$

$\mathbf{T}_{\mathbf{1 2}}$ - Dichlorovas 76 EC @ $2.63 \mathrm{ml} / 1$

$\underline{\mathbf{T}}_{\mathbf{1 3}}$ - Untreated control

$\overline{\mathbf{D A}} \mathbf{S}-$ Days after spraying 
Fig.2 Effect of insecticides on larval weight (g/larvae) on 5th day of 5th instar

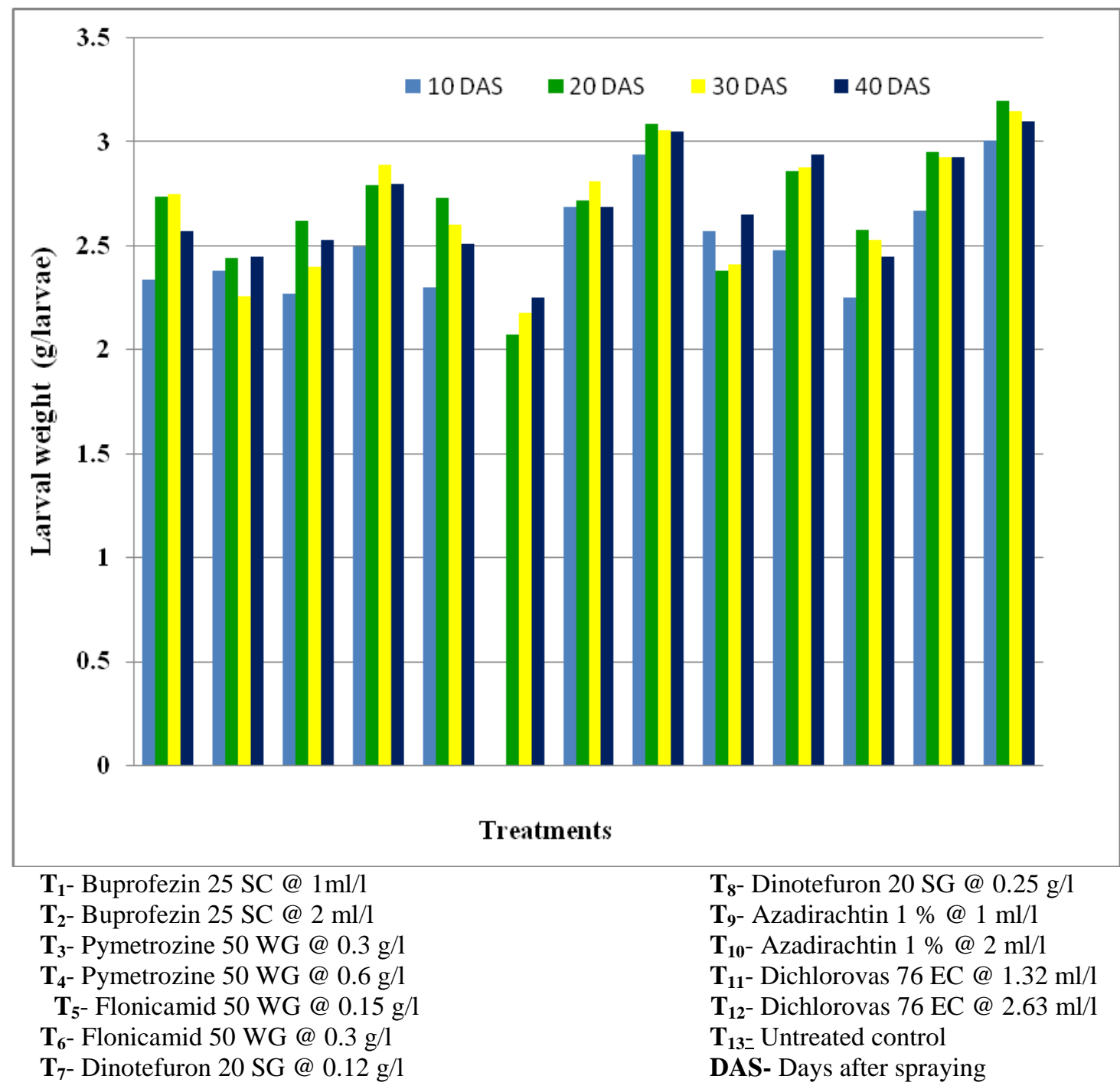

\section{Larval weight (g / larvae)}

The highest larval weight was recorded in untreated control (3.01 g/larva) which was on par with dinotefuron 20 SG @ $0.25 \mathrm{~g} / \mathrm{l}$ that exhibited larval weight of $2.94 \mathrm{~g} / \mathrm{larva}$ and the lowest value for larval weight was recorded in the batches treated with dichlorvos 76 EC @ $1.32 \mathrm{ml} / \mathrm{l}(2.25 \mathrm{~g})$ which was statistically on par with buprofezin 25 SC @ $2 \mathrm{ml} / 1(2.38$ g/larva), buprofezin $25 \mathrm{SC} @ 1 \mathrm{ml} / \mathrm{l}(2.34$ g/larva), flonicamid 50 WG $0.15 \mathrm{~g} / \mathrm{l}(2.30$ g/larva) and pymetrozine 50 WG @ $0.3 \mathrm{~g} / \mathrm{l}$ (2.27 g/larva) among the 10 DAS reared silkworms. In the silkworm batch treated with the mulberry leaves 20 DAS, the highest larval weight was recorded in untreated control (3.20 g/larva) which was statistically on par with dinotefuron 20 SG @ $0.25 \mathrm{~g} / \mathrm{l}$ (3.09 g/ larva) and the lowest larval weight was recorded in the batches treated with flonicamid 50 WG @ $0.3 \mathrm{~g} / \mathrm{l}(2.07 \mathrm{~g} / \mathrm{larva})$ which exhibited significant difference. There was significant difference among the treated batches on 30 DAS the mulberry leaves where the highest larval weight of $3.15 \mathrm{~g}$ per larva was exhibited in the untreated control which was on par with dinotrefuron 20 SG @ 0.25 g/l (3.06 g/larva) 
and dichlorvos 76 EC @ 2.63 ml/1 (2.93 g/ larva) whereas the lowest larval weight was recorded in the treatment flonicamid $50 \mathrm{WG}$ @ $0.3 \mathrm{~g} / \mathrm{l}$ (2.18 g/larva) and buprofezin $25 \mathrm{SC}$ @ $2 \mathrm{ml} / \mathrm{l}(2.26 \mathrm{~g} / \mathrm{larva})$ which were statistically on par. The silkworm batch treated with the mulberry leaves 40 DAS exhibited significant difference. The highest larval weight was recorded in untreated control (3.10 g/larva) which was significantly on par with dinotefuron 20 SG @ $0.25 \mathrm{~g} / 1$ (3.05 g/larva), azadiractin $1 \%$ @ 2 ml/l (2.94 g/larva) and dichlorvos 76 EC @ 2.63 ml/l (2.93 g/ larva) and the lowest larval weight was recorded in the batches treated with flonicamid 50 WG @0.3 g/l (2.25 g/ larva) (Table 6; Fig. 2).

Among the insecticide treatments, lowest larval weight was recorded in flocnicamid @ $0.3 \mathrm{~g} / \mathrm{l}$ and highest larval weight was recorded in dinotefuran 20 SG @ $0.25 \mathrm{~g} / 1$ and dichlorvos 76 EC @ $2.63 \mathrm{ml} / \mathrm{l}$ at 10, 20 30, 40 DAS. Larval weight in dinotefuran 20 SG @ $0.25 \mathrm{~g} / \mathrm{l}$ treatment was on par with that of untreated control. Among insecticides, dinotefuron application recorded higher larval weight. The dinotefuran belongs to neonicotinoid group of pesticides which is effective against sucking pests. Similar finding of increased larval weight upon application of neonicotinoid insecticide, thiamethoxam was reported by Manoja et al., (2011). Some insecticides are known to decrease the larval weight of silkworms fed with treated leaves. When mulberry leaves soaked in buprofezin and fed to silkworms, it resulted in significant decrease in the last instar larval weight in comparison to control (Maria et al., 2000)

\section{Effective rate of rearing (ERR) (\%)}

The silkworm batch reared by feeding mulberry leaves 10 DAS of insecticides exhibited significance difference in ERR percentage. The maximum ERR was observed in the treatment of pymetrozine 50 WG @ 0.3 $\mathrm{g} / \mathrm{l}(100.00 \%)$. Every other treatments were found statistically on par with each other except flonicamid 50 WG @ $0.3 \mathrm{~g} / 1$ (0) and $0.15 \mathrm{~g} / \mathrm{l}(87.00 \%)$. On $20^{\text {th }}$ DAS the silkworm batches reared exhibited non-significant results among the treatments. The maximum ERR was observed in the treatment dinotefuron 50 SG @ 0.25g/1 (97.77\%) and pymetrozine $50 \mathrm{WG} @ 0.6 \mathrm{~g} / \mathrm{l}$ recorded the lowest ERR of 96.67 per cent. ERR for 30 DAS was found non significant between the treatments. The treatment azardirachtin 1\% @ 2ml/l (100.00 ERR \%) and dichlorvos 76 EC @ $2.63 \mathrm{ml} / 1$ (100.00\%) recorded highest ERR $\%$, followed by buprofezin 25 SC @ 1ml/1 $(93.30 \%)$ and untreated control recorded the least ERR of 95.50 per cent. ERR for 40 DAS was also found non significant between treatments. Flonicamid 50 WG @ 0.3 g/l (98.87\%) and dichlorvos 76 EC @ 1.32 ml/1 (98.87\%) exhibited highest ERR and the least was seen in the treatment dinotefuron $50 \mathrm{SG}$ (a) $0.12 \mathrm{~g} / 1(94.43 \%)$.

ERR of 100 per cent was recorded in silkworms fed on mulberry leaves from treatments pymetrozine $50 \mathrm{WG} @ 0.3 \mathrm{~g} / \mathrm{l}$ at 10 DAS, azadirachtin 1\% @ $2 \mathrm{ml} / 1$ and dichlorvos 76 EC @ $2.63 \mathrm{ml} / \mathrm{l}$ at $30 \mathrm{DAS}$. However, there was no significant difference with regard to ERR per cent among the treatments at 20,30 and 40 DAS. At 10 DAS, lowest ERR of 87 per cent was recorded in flonicamid 50 WG @ 0.15g/l (Table 7).

Manoja et al., (2011) studied potential efficacy of new pesticides for the control of mulberry whitefly and its impact on silkworm rearing and they found that 0.015 per cent thiamethoxam, a neonicotinoid treated silkworms recorded a higher ERR of 94.35 per cent followed by dichlorvos @ $0.1 \%$ (92.96 $\%$ ERR). Similar observations were made with bioassay of $1 \%$ neem oil treated mulberry leaves resulted in highest ERR per cent $(93 \%$ 
ERR) among other insecticides viz., monocrotophos, acephate and dichlovos (Bandyopadhyay et al., 2013). Neem formulation recorded ERR at par with untreated control to the results noticed during the present investigation.

Contrarily, adverse effects of pesticide application on ERR per cent of silkworm Bombyx mori was reported by Gayathi. (2007). She reported that, when worms fed from fourth and fifth instar onwards at different days after spraying recorded minimum ERR of 39.07 per cent when treated with methyl demeton $(0.05 \%)$ and maximum ERR of 47.59 per cent was recorded with higher concentration of nimbicidin (6 ppm).

Among the various treatments, larval mortality was noticed only when worms were fed with leaves harvested at 10 DAS in the treatment flonicamid 50 WG @0.3 g/l(100\%) and no larval mortality was noticed in any of the insecticide treatments when fed with leaves harvested at 20, 30 and 40 DAS. Though flonicamid is a selective feeding blocker specific to sucking insects with repellent action, it appears that silkworms have lower tolerance limits for this molecule. With most of the insecticides, the prolongation of larval period was noticed at third, fourth and fifth instars when they were fed with insecticide treated leaves harvested at different days after spray. The third instar larval duration of silkworm was prolonged to $84.70 \& 84.37 \mathrm{~h}$ when the worms were fed on mulberry leaves sprayed with flonicamid 50WG @ $0.3 \mathrm{~g} / \mathrm{l}$ with leaves harvested 10DAS and the shortest larval duration was observed in the treatment dinotefuron 50SG @ $0.25 \mathrm{~g} / \mathrm{l}$ followed by dichlorvos 76 EC @ 2.63 ml/l. This may be due to the later effects of trace amount of insecticides in the leaves. Among the insecticide treatments, the highest larval weight was recorded in dinotefuran 20 SG @ $0.25 \mathrm{~g} / \mathrm{l}$ which was on par with that of untreated control, as well as dichlorvos $76 \mathrm{EC}$ @ $2.63 \mathrm{ml} / 1$ and lowest larval weight was recorded in flocnicamid @ $0.3 \mathrm{~g} / 1$ at 10, 20 30, 40 DAS as dinotefuran belongs to neonicotinoid group of pesticides. By considering these facts dinotefuron 20SG can be used as an effective substitute for DDVP in Sericulture.

\section{References}

Bandyopadhyay, U. K., Chatterjee, S. Maji, C. and Bindroo, B. B., 2013. Efficacy of plant oils against leaf webber (Glyphodes pyloalis Walker) on Mulberry (Morus alba L.). Annals of Plant Protection Sciences 44(8): 49-53. Bandyopadhyay, U.K., Santhakumar, M.V. and Saratchandra, B., 2005. Role of insecticides and botanicals in regulating whitefly (Dialeuropora decempuncta) incidence and their influence on some economic traits of silkworm (Bombyx mori L.). Annals of Plant Protection Sciences 13 (1): 4853.

Dandin, S.B., Jayaswal, J. and Giridhar, K., 2003. Handbook of Sericulture Technologies. CSB, Bangalore. P.287.

Gayathi, M., 2007. Studies on tukra and powdery mildew diseases in mulberry and their impact on the growth and development of silkworm, B. Mori. M.Sc. (Agri.) Thesis, ANGRAU Bapatla P.42-112.

Ghosh, A., Samantha, A. and Chatterjee, M.L., 2014, Dinotefuran, a third generation neonicotinoid insecticide for management of rice brown planthopper. African.J.Agric.Res., 9(8): 750-754.

Kumutha, P., Padmalatha, C. and Ranjit singh, A. J. A., 2013. Effect of pesticides on the reproductive performance and longevity of Bombyx mori L. International Journal of Current 
Microbiology and Applied Sciences 2(9):74-78.

Kumutha, P., Padmalatha, C., Sam Manohar Doss, S. and Aranjit singh, A. J. A., 2009. Toxicity evaluation of neem oil and metacid on the development of the silkworm, Bombyx mori L. Uttar Pradesh Journal of Zoology 29(4) :220-222.

Li,H.Y., Huang,W. J, Xie, J. L,Qin Bao, B. Q. and We, R., 1995. Thee organophosphate insecticides on mulberry thips control and their residual toxicities on silkworm. Acta Sericologica Sinica. 03(1):698-700.

Mahui , Wang Kaiyun, Wang Hongyan. and Sishuding., 2006. Selective toxicity of chlorfenapyr between mulberry silkworms and mulberry pests. Acta Entomologica Sinica, 49(4):599-603.

Manoja, P., Bhattacharya, D. K., Kar, B., Das, N. K., Saha, A. K. and Bindroo, B. B., 2011. Potential efficacy of new pesticides for the control of mulberry whitefly and its impact on silkworm rearing. Journal of Plant Protection and Science 3(1) : 57-60.

Maria, E. V., Paschalis, C.H. and Sergios, K., 2000. Effects of Applaud on the growth of silkworm (Lepidoptera: Bombycidae). Journal of Economic Entomology 93(2): 290-292.

Narasimhanna, M. N., 1988. Manual on silkworm egg production. CSB, Bangalore, pp: 146.

Narayanaswamy, K. C., Harish, B.S. and Jagadish, K. S., 2017. Effect of NSKE and IPM module treated leaves on rearing performance of the silkworm, Bombyx mori L. Mysore Journal of Agricultural Sciences 51(1): 102-107.

Sik, K., Ryong, S. H. and Sang, K. R., 1976. Study of various pollutions on silkworm rearing in autumn. Sericulture Journal of Korea 18(3):1719.

Yokoyama, T., 1962. Synthesized Science of Sericulture. Japan, pp. 39-46.

\section{How to cite this article:}

Yeshika, M.P., K.G. Banuprakash, K. Muruli Mohan and Vinoda, K.S. 2019. Effect of Novel Insecticide Molecules in Mulberry on Larval Parameters of Silkworm Bombyx mori L. Int.J.Curr.Microbiol.App.Sci. 8(11): 1112-1125. doi: https://doi.org/10.20546/ijcmas.2019.811.131 